\title{
Observing Plasmonic-Molecular Resonance Coupling on Single Gold Nanorods
}

\author{
Weihai Ni, ${ }^{\dagger}$ Tobias Ambjörnsson, ${ }^{\ddagger}$ Sten Peter Apell, ${ }^{\S}$ Huanjun Chen, ${ }^{\dagger}$ and Jianfang Wang ${ }^{*}{ }^{\dagger}$ \\ ${ }^{\dagger}$ Department of Physics, the Chinese University of Hong Kong, Shatin, Hong Kong SAR, China, ${ }^{*}$ Department of \\ Theoretical Physics, Lund University, Sölvegatan 14A, SE-223 62 Lund, Sweden, and ${ }^{\S}$ Department of Applied \\ Physics, Chalmers University of Technology, SE-412 96 Göteborg, Sweden
}

\begin{abstract}
Strong plasmonic-molecular resonance coupling occurs between noble metal nanocrystals and organic adsorbates when the plasmonic resonance is degenerate with the molecular one. This interaction forms the basis for many fundamental studies and practical applications. We describe here the first direct measurement of the resonance coupling on single gold nanorods. The darkfield scattering technique is employed. The nanorods are embedded in hydrogel to facilitate uniform dye adsorption. The adsorbed dye molecules exhibit both monomer and H-aggregate absorption bands. The same gold nanorods are measured before and after the dye adsorption. Both strong and weak coupling are investigated by selecting nanorods with different longitudinal plasmon bands. Excellent agreement between the experiments and an analytic theory is obtained. The resonance coupling reveals a unique threeband structure. The tunability of the coupling on individual nanorods is further demonstrated by photodecomposing the adsorbed dye molecules.
\end{abstract}

KEYWORDS Absorption, dark-field scattering, gold nanorods, resonance coupling, surface plasmon

I nteractions between noble metal nanocrystals and organic adsorbates give rise to very interesting phenomena, such as plasmon-enhanced fluorescence, ${ }^{1-6}$ fluorescence quenching, ${ }^{7,8}$ plasmon resonance energy transfer, ${ }^{9}$ and molecular-plasmonic resonance coupling. ${ }^{10-20}$ Strong plasmonic-molecular resonance coupling occurs when the plasmonic resonance is degenerate with the molecular resonance. This interaction forms the basis for amplifying optical signals, fabricating optical devices, and detecting biological molecules and processes. Previous studies on the resonance coupling have been limited on ensemble metal nanocrystals, ${ }^{10,11,13,15,17,18}$ where ensemble averaging impedes a thorough study. Here, we report on the first direct measurement of the resonance coupling on single gold nanorods. We employ the dark-field scattering technique ${ }^{21}$ and use Au nanorods that are embedded in hydrogel matrices to facilitate the uniform adsorption of dye molecules. Through electrostatic interactions, the dye molecules are adsorbed uniformly on the Au nanorods that are embedded in hydrogel matrices. The adsorbed dye molecules exhibit both monomer and H-aggregate absorption bands. The same Au nanorods are precisely investigated before and after the dye adsorption. Both strong and weak resonance coupling have been studied by selecting nanorods with different longitudinal plasmon resonance energies, and excellent agreement between the experimental results and an analytic theoretical framework has been obtained by including the two molecular absorption bands in the theory.

* To whom correspondence should be addressed. E-mail: jfwang@phy.cuhk.edu.hk Tel: +852 3163 4167. Fax: +8522603 5204.

Received for review: 09/1/2009

Published on Web: 00/00/0000
The coupling reveals a unique three-band structure in the energy diagram. The tunability of the resonance coupling has further been demonstrated by photodecomposing the dye molecules adsorbed on individual Au nanorods.

Spatially isolated Au nanorods were embedded in agarose gel films (Figure 1a). The use of Au nanorods is because the longitudinal plasmon resonance energy of each nanorod is determined by the length-to-diameter aspect ratio, and they exhibit reduced plasmon damping compared to spherical Au nanocrystals. ${ }^{21}$ Agarose gel has a three-dimensionally interconnected porous network. Its pore size is on the order of a few hundred nanometers, and its pore wall is highly hydrophilic. Agarose gel is therefore an ideal matrix for the immobilization of Au nanorods. It can not only facilitate single-particle dark-field scattering measurements but also provide a highly flexible and chemically accessible environment for physical and chemical processes to occur at specific local positions.

The Au nanorods were grown in aqueous solutions using a seed-mediated method. ${ }^{22}$ The as-grown Au nanorods are stabilized with cetyltrimethylammonium bromide (CTAB) surfactants. Anisotropic oxidation ${ }^{23}$ and transverse overgrowth ${ }^{24}$ were employed to finely tailor the longitudinal plasmon resonance wavelengths of the nanorods within the range of 600-950 $\mathrm{nm}$. Figure $1 \mathrm{~b}$ shows a transmission electron microscopy (TEM) image of a representative nanorod sample that exhibits an ensemble longitudinal plasmon resonance wavelength at $685 \mathrm{~nm}$. The nanorods are cylindrical, and their sizes are highly uniform. HITC dye (Figure 1c) was chosen to form organic-inorganic hybrid structures with the Au nanorods. When dissolved in water, HITC exhibits a major absorption peak at $736 \mathrm{~nm}$ with an absorp- 

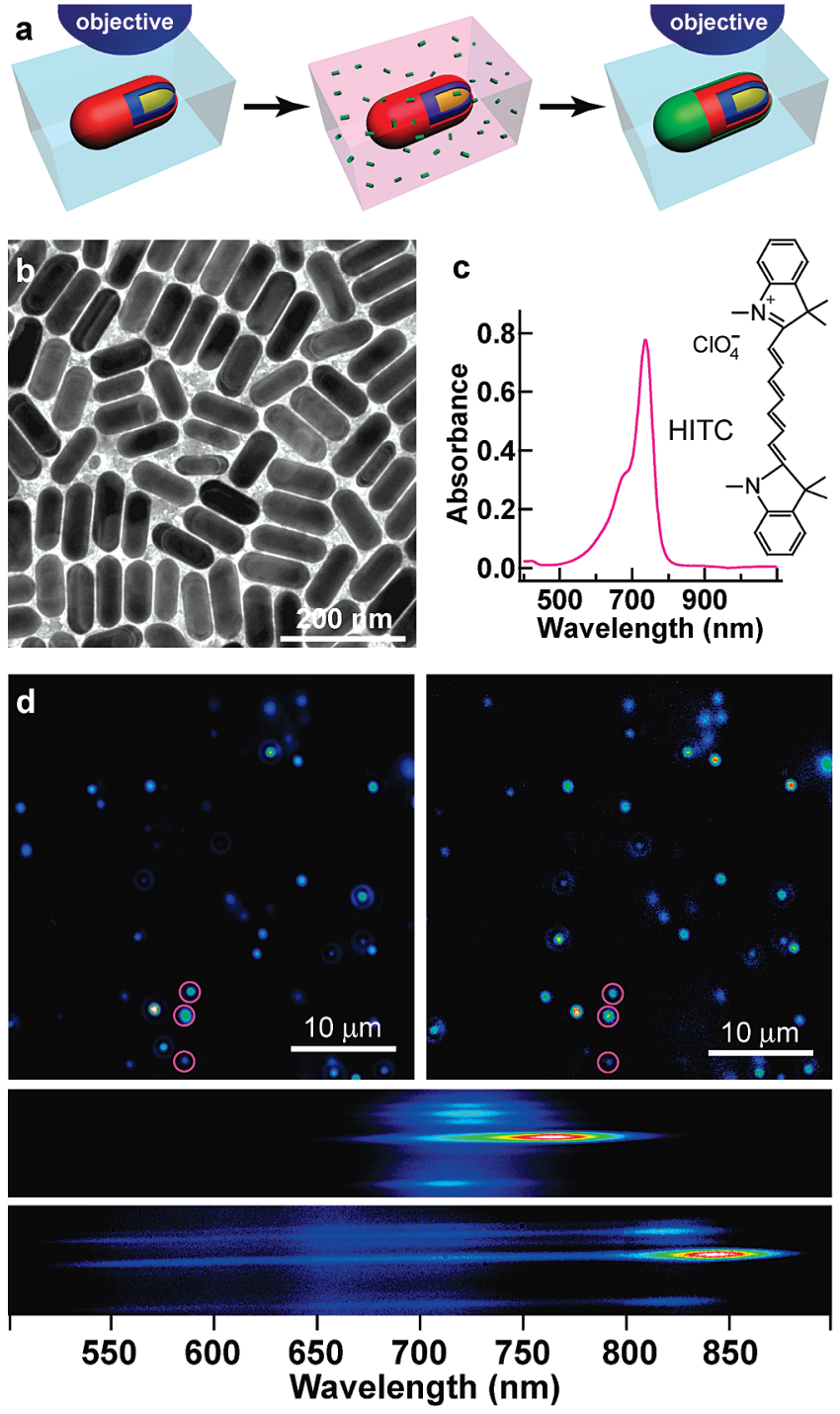

FIGURE 1. Observation of plasmonic-molecular resonance coupling on single Au nanorods embedded in agarose gel films. (a) Schematic illustration of the experimental procedure. The Au nanorods were first embedded in an agarose gel film. The gel film was then immersed in an ethanolic solution of HITC and subsequently immersed in water. (b) TEM (FEI CM120) image of a typical as-grown Au nanorod sample with an average diameter, length, and aspect ratio of $31 \pm 4 \mathrm{~nm}, 87 \pm 8 \mathrm{~nm}$, and $2.8 \pm 0.4$, respectively. (c) Absorption spectrum of HITC in water at $0.01 \mathrm{mM}$ and its molecular structure. The optical path length for the absorption spectral measurement (Hitachi U-3501 UV/visible/NIR spectrophotometer) is $0.5 \mathrm{~cm}$. (d) Scattering and spectral images taken from the same region in a gel film before and after HITC adsorption. Upper left and right: scattering images before and after HITC adsorption, respectively. Middle and bottom: spectral images of the three marked nanorods before and after HITC adsorption.

tion coefficient of $1.56 \times 10^{5} \mathrm{M}^{-1} \mathrm{~cm}^{-1}$ and a vibrational shoulder at $678 \mathrm{~nm}$. The major peak represents the dye's $\mathrm{M}_{\mathrm{s}}$ band, corresponding to its monomeric unperturbed solution state. ${ }^{25}$ Each HITC molecule carries a positive charge. Because the CTAB-stabilized Au nanorods are also positively charged, a layer of poly(sodium 4-styrenesulfonate) (PSS, $\mathrm{MW}=70000$ ) is adsorbed on the nanorod surface through electrostatic interactions to make the nanorods negatively charged. ${ }^{26}$ The particle concentration in the resultant PSS-coated nanorod solution was estimated to be $2 \mathrm{nM}$.

For the embedding of the PSS-coated Au nanorods into agarose gel films, the nanorod solution was diluted by 40 times to $0.05 \mathrm{nM}$. A $200 \mu \mathrm{L}$ portion of the diluted nanorod solution was added in a glass beaker containing $4 \mathrm{mg}$ of agarose and $1.8 \mathrm{~mL}$ of water. The beaker was placed in a water bath at $80^{\circ} \mathrm{C}$. The mixture solution became clear after being stirred mildly for a few minutes. Twenty microliters of the resultant agarose gel solution was dropcast on a clean glass slide of $0.16-0.19 \mathrm{~mm}$ thickness. Another glass slide with engraved alignment marks was then carefully placed on the top, with two small glass slides inserted in between. An agarose gel film with a thickness of $0.16-0.19 \mathrm{~mm}$ was thus obtained. It was allowed to cool down naturally. The obtained gel film is transparent. Extinction spectral measurements reveal that the encapsulated nanorods are uniformly distributed without aggregation (Figure S1, Supporting Information). The gel film was kept wet throughout all the measurements.

The scattering of the individual Au nanorods that were embedded in the agarose gel films was measured with the dark-field technique. The measurements were carried out on an Olympus BX60 optical microscope integrated with an Acton SpectraPro 2300i monochromator and a Princeton Instruments Pixis 512B charge-coupled device camera, which was thermoelectrically cooled to $-50{ }^{\circ} \mathrm{C}$. The white light from a $100 \mathrm{~W}$ quartz-halogen-tungsten lamp was passed through an oil-immersion dark-field condenser (NA $=1.2-1.4$ ) and illuminated on the gel film (Figure S2, Supporting Information). The scattered light was collected by a long working distance objective (LMPlan-IR, 50×, NA $=0.55$ ) and reflected to the entrance slit of the monochromator. The scattering spectra from individual nanorods were corrected by subtracting the background spectra taken from the adjacent regions without Au nanorods and dividing with the calibrated response curve of the entire optical system.

After the scattering of the PSS-coated Au nanorods was measured, the oil residue left on the glass slide from the use of the dark-field condenser was carefully removed with a wipe soaked with ethanol in order to minimize contamination. For HITC adsorption, the gel film was first immersed in an ethanolic $0.1 \mathrm{mM}$ solution of HITC overnight and then in water for $30 \mathrm{~min}$. The film was taken out, and excess water on the glass slides were removed with filter paper. The use of ethanol is because the solubility of HITC in ethanol is much larger than that in water and the diffusion of HITC in the agarose gel in an ethanolic environment is much faster than that in an aqueous environment. The final immersion of the gel film in water was to allow for the exchange of ethanol with water and the adsorption of HITC molecules on the PSS-coated Au nanorods through electrostatic interactions. The adsorption of positively charged dye molecules onto the negatively charged, PSS-coated nanorod surface 
was confirmed by the quenching of the fluorescence from adsorbed dye molecules (Figure S3, Supporting Information). The large pores in the gel film facilitate the formation of a uniformly adsorbed layer of HITC on the Au nanorods. In contrast, the deposition of the Au nanorods on planar substrates would preclude the adsorption of a uniform dye layer owing to the charged substrate surface and the intimate contact between the nanorods and the substrates.

The resultant hybrid nanostructures embedded in the gel film were then subjected to the dark-field scattering measurements again. The Au nanorods that were imaged before HITC adsorption were located thereafter by first moving the sample stage horizontally according to the alignment marks engraved on the glass slides and then moving vertically by the same depth as before HITC adsorption (Figure S4, Supporting Information). The distribution patterns of the bright spots on the dark-field images acquired before and after HITC adsorption were compared to finely adjust the position of the sample stage. The lamp intensity and the exposure time were kept the same so that the scattering intensities from the same Au nanorods can be compared. Figure $1 \mathrm{~d}$ shows two representative scattering images of the embedded Au nanorods that were taken before and after HITC adsorption. Each bright spot represents a single Au nanorod, as corroborated by their scattering spectra. The scattering intensity exhibits a large variation, which is mainly caused by the variation of the depth of the nanorods in the agarose gel film. The distribution patterns of the bright spots on both images match each other very well. A few spots are seen to show changes in their intensities or positions. We believe they correspond to the nanorods that are situated within the large pores. The positions of these nanorods are slightly displaced during the solvent exchange. These nanorods are excluded from our analysis.

The scattering spectral images of the Au nanorods before and after HITC adsorption were measured and compared (Figure $1 d$, middle and bottom). The plasmon bands of the nanorods exhibit distinct changes after HITC adsorption. In order to see clearly the HITC adsorption-induced changes, scattering spectra were extracted from the spectral images. One example is shown in Figure 2a. The PSS-coated nanorod exhibits a scattering peak at $725 \mathrm{~nm}$, which arises from the longitudinal plasmon resonance mode of the nanorod. After HITC adsorption, the peak splits into three peaks, which are at 548,727, and $817 \mathrm{~nm}$, respectively. The occurrence of three scattering peaks indicates that the plasmonic resonance of the nanorod and the molecular resonances of HITC are strongly hybridized. Such a resonance coupling behavior has not been observed in previous ensemble experiments. ${ }^{10,11,13,15,17,18}$

Because the resonance coupling behavior is highly dependent on the spectral overlap between the plasmonic and molecular resonance bands, HITC molecules were also adsorbed on a PSS-coated glass substrate to model their adsorption on the PSS-coated Au nanorods so that the

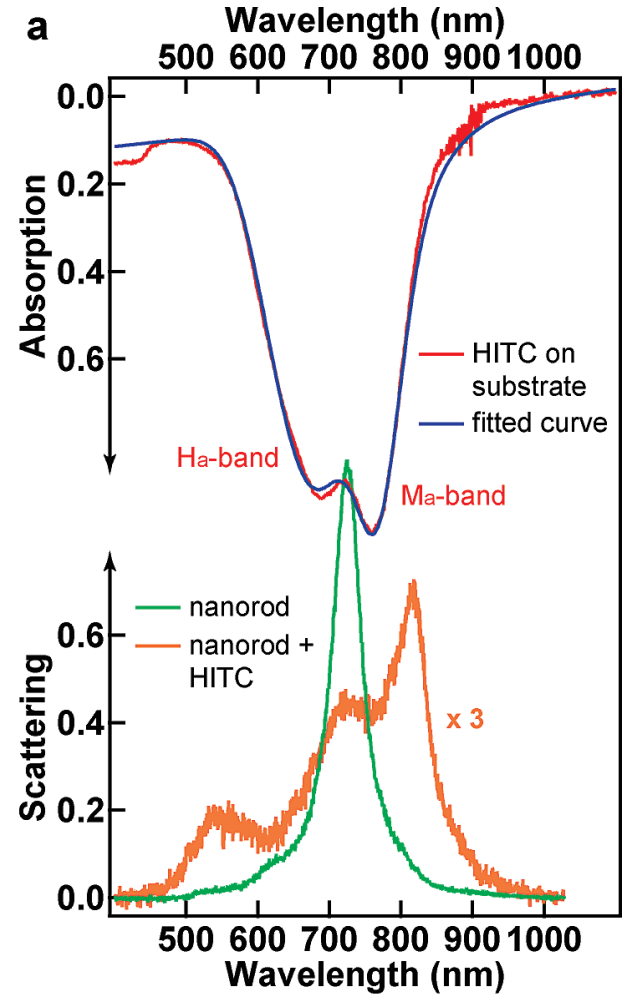

b
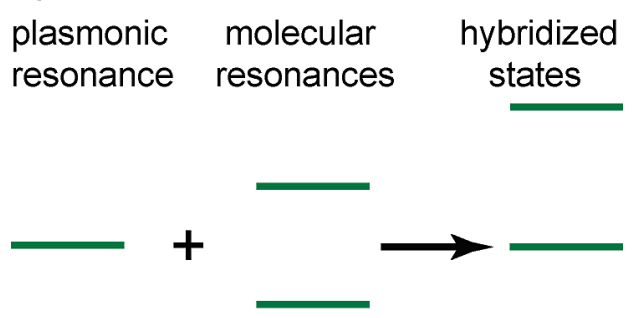

C

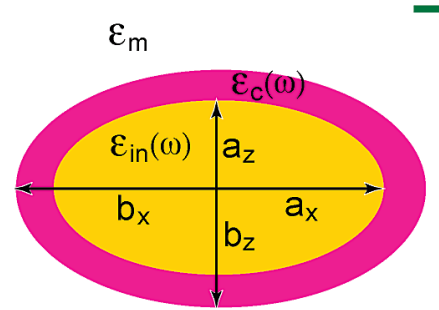

FIGURE 2. Plasmonic-molecular resonance coupling. (a) Illustration of the resonance coupling. Upper: measured (red) and fitted (blue) absorption spectra of HITC on a PSS-coated glass slide. Lower: scattering spectra of a single Au nanorod before (green) and after (orange) HITC adsorption. (b) Schematic energy diagram showing the coupling-induced formation of three hybridized states. (c) Schematic model of a nanorod-dye hybrid structure.

absorption spectrum of the adsorbed HITC molecules could be measured. The used glass slides were cleaned and functionalized by immersion in an ethanolic $10 \mathrm{vol} \%$ solution of (3-aminopropyl)triethoxysilane for $30 \mathrm{~min}$. After being rinsed thoroughly with water, the amine-functionalized, positively charged glass slides were immersed in an aqueous $2 \mathrm{~g} \mathrm{~L}^{-1}$ solution of PSS that also contained $6 \mathrm{mM}$ of 
$\mathrm{NaCl}$ under mild stirring for $3 \mathrm{~h}$. The PSS-coated slides were rinsed with water again and then immersed in an aqueous $0.07 \mathrm{mM}$ HITC solution. The slides were kept in the HITC solution for $30 \mathrm{~min}$, followed by several times of rinsing with water to remove excess HITC molecules. The slides were subsequently placed under water in a glass beaker. Each of them was covered with two clean glass slides on both sides. As a result, the HITC layers adsorbed on both sides were sandwiched between two glass slides and maintained in an aqueous environment. A reference sample for the absorption measurements was also made by stacking together three glass slides under water.

Figure 2a shows the absorption spectrum of the HITC molecules adsorbed on the PSS-coated glass slide. Two pronounced absorption peaks are observed. One is slightly red-shifted relative to the $\mathrm{M}_{\mathrm{s}}$ band. It is ascribed to the dye's $\mathrm{M}_{\mathrm{a}}$ band, corresponding to the adsorbed monomers. ${ }^{25}$ The red shift is commonly interpreted as being caused by the increase in the average surrounding refractive index when the dye monomers are adsorbed from solutions to substrates. The other one is at a higher energy than the $\mathrm{M}_{\mathrm{a}}$ band and is comparable in intensity with the $M_{a}$ band. The appearance of this rather broad peak indicates the formation of $\mathrm{H}$-aggregates with different sizes on the substrate. In $\mathrm{H}$-aggregates, dye molecules are parallel-stacked. The angle between the line-of-centers of a column of the dye molecules and the long axis of any one of the parallel molecules is larger than $\sim 32^{\circ}$. The absorption peak arising from the $\mathrm{H}$-aggregates is usually known as the $\mathrm{H}_{\mathrm{a}}$ band. ${ }^{27}$ The unprecedented resonance coupling induced formation of the three hybridized states occurs only when the longitudinal plasmon band of the Au nanorod is in between the $\mathrm{M}_{\mathrm{a}}$ and $\mathrm{H}_{\mathrm{a}}$ band of HITC, as shown schematically in Figure 2b.

The dark-field spectroscopic measurements were further performed both before and after HITC adsorption on the single embedded Au nanorods that have varying longitudinal plasmon resonance wavelengths. Figure 3 shows the results for eight representative Au nanorods, with their longitudinal plasmon resonance wavelengths increasing from top to bottom. The intensities of the scattering spectra obtained after HITC adsorption have been normalized against the corresponding peak intensities of the spectra recorded before HITC adsorption. Compared to the corresponding PSS-coated Au nanorods, the nanorod-HITC hybrid nanostructures exhibit distinctly different scattering spectra, suggesting that the plasmonic and molecular resonances are strongly coupled together. The peak position and intensity induced by the resonance coupling vary systematically as a function of the original longitudinal plasmon resonance wavelength. When the longitudinal plasmon band is on the blue side of and close to the dye's $\mathrm{H}_{\mathrm{a}}$ band, the resultant peak is found blue-shifted and broadened, with a largely reduced intensity. As the plasmon band moves closer to the $\mathrm{H}_{\mathrm{a}}$ band, the coupling-induced peak becomes even weaker. When the plasmon band is in between the $\mathrm{H}_{\mathrm{a}}$ and $\mathrm{M}_{\mathrm{a}}$ band, the reso-

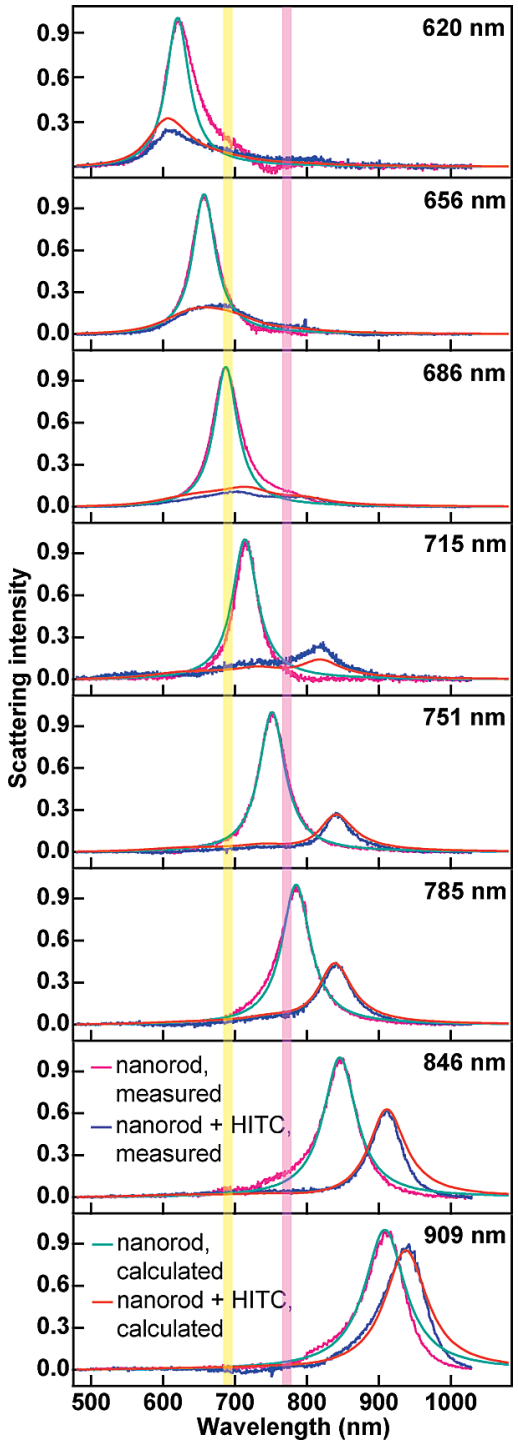

FIGURE 3. Dependence of the plasmonic-molecular resonance coupling behavior on the plasmon resonance wavelength of the $\mathrm{Au}$ nanorods. The experimental and theoretical scattering spectra of the single Au nanorods and the corresponding nanorod-HITC hybrid nanostructures are shown. The plasmon wavelengths of the Au nanorods before HITC adsorption are given at the top right corner of each plot. The yellow and pink vertical lines represent the $\mathrm{H}_{\mathrm{a}}$ and $M_{a}$ band of HITC, respectively. The scattering spectra have been normalized against the scattering peak maximum of each $\mathrm{Au}$ nanorod before HITC adsorption. In the theoretical modeling, the effective oscillator strength $c_{0}$ for the adsorbed HITC layer is set to be 2.3. The effective dye layer thickness $d$ is determined from the experimental scattering spectra to be 1.0, 0.9, 1.0, 1.4, 1.5, 0.9, 1.5, and $0.7 \mathrm{~nm}$ from top to bottom, respectively.

nance coupling produces three peaks, with one on the blue side of the $\mathrm{H}_{a}$ band, one in between the $\mathrm{H}_{\mathrm{a}}$ and $\mathrm{M}_{\mathrm{a}}$ band, and the third one lying on the red side of the $M_{a}$ band. The measured wavelength difference between the red-shifted peak and the original plasmon band reaches up to $105 \mathrm{~nm}$. As the plasmon band moves across the $\mathrm{M}_{\mathrm{a}}$ band and further to the red side of the $\mathrm{M}_{\mathrm{a}}$ band, the coupling produces a redshifted peak with a slightly reduced intensity. As the plasmon band moves even further to the red side of the $M_{a}$ band, both 
the red shift and intensity reduction get smaller, suggesting that the coupling becomes weaker.

Analytic theories have been developed to understand the resonance coupling in the hybrid systems of dye aggregates adsorbed on noble metal nanospheres, ${ }^{16}$ nanoshells, ${ }^{18}$ and prolate spheroids. ${ }^{28,29}$ Our results represent the first singleparticle measurement of the resonance coupling induced hybridization and pronounced plasmon shift. The singleparticle measurement allows us to make a direct comparison between the experimental results and theoretical calculations. The theory is modified from that developed for a prolate spheroid with a dye-coating layer that possesses a single molecular resonance band. ${ }^{28,29}$ We first consider the molecular resonances of the HITC adsorbate and the plasmon resonances of the PSS-coated Au nanorods. The essential parameters of both molecular and plasmonic resonances are extracted by comparing the calculated spectra with the experimental ones. These parameters are then fed into the formulas of the coupled system to calculate the coupling-induced scattering spectra.

The two molecular resonances of the HITC adsorbate on the PSS-coated Au nanorods, $\omega_{10}$ and $\omega_{20}$, which correspond to the dye's $M_{a}$ and $H_{a}$ band, are considered first. Two broadened Lorentzian functions are introduced to represent the two molecular resonances. The dye monomers and $\mathrm{H}$-aggregates are assumed to have isotropic transition dipole moment orientations (Figure 2c), owing to the flexibility of the PSS chains and the electrostatic interactions between the PSS chains and the dye molecules. The isotropic effective molecular polarizability can be described with

$$
\begin{aligned}
& \gamma(\omega)=\gamma_{\infty}+ \\
& \left(\gamma_{0}-\gamma_{\infty}\right)\left[C_{1} \int_{-\infty}^{\infty} G\left(\omega_{10}, \bar{\omega}_{10}, \sigma_{1}\right) \tilde{\gamma}\left(\omega, \omega_{10}, \Gamma_{1}\right) \mathrm{d} \omega_{10}+\right. \\
& \left.C_{2} \int_{-\infty}^{\infty} G\left(\omega_{20}, \bar{\omega}_{20}, \sigma_{2}\right) \tilde{\gamma}\left(\omega, \omega_{20}, \Gamma_{2}\right) \mathrm{d} \omega_{20}\right]
\end{aligned}
$$

Here, $\gamma_{\infty}$ is the high-frequency polarizability of the molecule and $\gamma_{0}$ is the static polarizability. $\gamma_{0}-\gamma_{\infty}$ is given by $\gamma_{0}-\gamma_{\infty}$ $=c_{0} v_{0} / 4 \pi$, with $c_{0}$ being the effective oscillator strength and $v_{0}$ being the unit cell volume per molecule. ${ }^{30}$ In eq 1 , the two functions under each integral are given by

$$
\begin{gathered}
\tilde{\gamma}(\omega, z, \Gamma)=\frac{z^{2}}{z^{2}-\omega^{2}-i \omega \Gamma} \\
G(\omega, \bar{\omega}, \sigma)=\frac{1}{\sqrt{2 \pi \sigma^{2}}} \exp \left[-\frac{(\omega-\bar{\omega})^{2}}{2 \sigma^{2}}\right]
\end{gathered}
$$

In eq 3 , a Gaussion distribution for $\omega_{10}$ and $\omega_{20}$ is assumed, effectively modeling the disorder in the arrangement of the dye molecules on the surface. $\sigma$ is the peak width. The parameters $C_{1}$ and $C_{2}$ are the weighting factors of the two molecular resonances. The condition that $\gamma(\omega \rightarrow 0)=\gamma_{0}$ requires $C_{1}+C_{2}=1$. Equation 1, together with a linear background to account for the dye absorption in the UV region, is used to fit the absorption spectrum of HITC adsorbed on the PSS-coated substrates (Figure 2a). The following parameters for the two molecular resonances of the HITC adsorbate are obtained from fitting: $\hbar \bar{\omega}_{10}=1.61$ $\mathrm{eV}(772 \mathrm{~nm}), \hbar \bar{\omega}_{20}=1.80 \mathrm{eV}(690 \mathrm{~nm}), \hbar \Gamma_{1}=\hbar \Gamma_{2}=0.012$ $\mathrm{eV}, \hbar \sigma_{1}=0.06 \mathrm{eV}, \hbar \sigma_{2}=0.19 \mathrm{eV}, C_{1}=0.17$, and $C_{2}=0.83$. The subscripts 1 and 2 represent the $\mathrm{M}_{\mathrm{a}}$ and $\mathrm{H}_{\mathrm{a}}$ band of HITC, respectively. The broadening of the $\mathrm{H}_{\mathrm{a}}$ band indicates that there is a large variation in the number of HITC molecules in each $\mathrm{H}$ aggregate.

We next consider the electromagnetic response of a bare Au prolate spheroid, which is used to model a PSS-coated $\mathrm{Au}$ nanorod. The omission of the PSS and CTAB layers is reasonable because they are very thin and exhibit no molecular resonances near the plasmon resonances of the nanorods. In addition, Au prolate spheroids have been shown to be a very good approximation in modeling the absorption and scattering properties of Au nanorods. ${ }^{31}$ The aspect ratio of the Au spheroid is defined as $R=a_{x} / a_{z}$, with $a_{v}$ being the principal semiaxis along the direction $v(v=x$, $y$, or $z$ ) and $a_{x} \geq a_{y}=a_{z}$ (Figure 2c). Within the quasi-static approximation, the polarizability of the spheroid, which has a dielectric function $\varepsilon_{\text {in }}$ and is embedded in a surrounding medium of a dielectric constant $\varepsilon_{\mathrm{m}}$, is given by ${ }^{29}$

$$
\alpha_{v v}=\frac{V}{4 \pi n_{\nu}} \frac{\varepsilon_{\text {in }}-\varepsilon_{\mathrm{m}}}{\varepsilon_{\text {in }}+\varepsilon_{\mathrm{m}}\left(1 / n_{v}-1\right)}
$$

Here, $V=4 \pi a_{x} a_{y} a_{z} / 3$ is the volume of the spheroid and $n_{v}$ is the standard depolarization factor along the $v$ direction. The Drude model is employed for the dielectric function of gold

$$
\varepsilon_{\text {in }}(\omega)=\varepsilon_{\infty}-\frac{\omega_{\mathrm{p}}{ }^{2}}{\omega\left(\omega+i \Gamma_{\mathrm{p}}\right)}
$$

Here, $\varepsilon_{\infty}$ is the dielectric constant of gold at large frequencies, $\omega_{\mathrm{p}}$ is the s-electron plasmon frequency, and $\Gamma_{\mathrm{p}}$ is the Drude damping constant. $\Gamma_{\mathrm{p}}$ is composed of three contributions for the Au nanorods

$$
\Gamma_{\mathrm{p}}=\Gamma_{\text {bulk }}+\Gamma_{\text {rad }}+\Gamma_{\text {surf }}
$$

Here, the three terms represent the contributions to the damping constant from bulk gold, radiation, and surface scattering, respectively. The scattering cross section for the excitation polarization along the $v$ direction of the prolate spheroid is given by

$$
\sigma_{v}^{\text {scat }} \propto k^{4}\left(\left|\alpha_{v v}\right|^{2}\right)
$$

In our calculations, $\varepsilon_{\mathrm{m}}=1.77$ is used for water in the surrounding medium of the Au nanorods. For gold, $\hbar \omega_{\mathrm{p}}=$ $8.95 \mathrm{eV}, \varepsilon_{\infty}=9.5$, and $\hbar \Gamma_{\text {bulk }}=0.069 \mathrm{eV}$. The aspect ratio $R$, in the range of $1-8$, is determined by the measured longitudinal plasmon resonance wavelengths of the PSScoated Au nanorods. The additional damping, $\Gamma_{\text {rad }}+\Gamma_{\text {surf, }}$ is set to be proportional to $\Gamma_{\text {bulk }}$ with a proportionality constant of $0.4-0.8$. The calculated scattering spectra match the experimental ones very well (Figure 3 ).

The analytic theory for the dipolar polarizability of a hybrid plasmonic-molecular coupling system considers a prolate spheroid with a dye-coating layer (Figure 2c). In the experiments, the dye layer is not in direct contact with the nanorod surface owing to the presence of the CTAB and PSS 
layers. The spacing has previously been measured to be 1.4 $\mathrm{nm} .{ }^{17}$ This spacing is not considered in our modeling. The principal semiaxes of the inner and outer spheroids are $a_{v}$ and $b_{v}$ with $a_{x} \geq a_{y}=a_{z}$ and $b_{x} \geq b_{y}=b_{z}$, respectively. The dye coating thickness is $d$. In the original theory, a dye layer with anisotropic transition dipole moment orientations is considered. ${ }^{29}$ Because the transition dipole moments associated with the HITC coating layer in our experiments are isotropic, the polarizability of such a system is given by ${ }^{32}$

$$
\begin{gathered}
\left(\varepsilon_{0}-1\right)\left[\varepsilon_{0}\left(1-1 / n_{v}{ }^{\mathrm{i}}\right)-\varepsilon_{i}\right]- \\
\alpha_{v v}=\frac{V_{0}}{4 \pi n_{v}{ }^{\circ}} \frac{\rho_{v}\left[\varepsilon_{0}\left(1-1 / n_{v}{ }^{\circ}\right)-1\right]\left(\varepsilon_{0}-\varepsilon_{i}\right)}{\left(\varepsilon_{0}+1 / n_{v}{ }^{\circ}-1\right)\left[\varepsilon_{0}\left(1-1 / n_{v}{ }^{\mathrm{i}}\right)-\varepsilon_{i}\right]-} \\
\rho_{v}\left[\varepsilon_{0}\left(1-1 / n_{v}{ }^{\circ}\right)+1 / n_{v}{ }^{\circ}-1\right]\left(\varepsilon_{0}-\varepsilon_{i}\right)
\end{gathered}
$$

Here, $V_{0}=4 \pi b_{x} b_{y} b_{z} / 3, \varepsilon_{i}=\varepsilon_{\text {in }} / \varepsilon_{\mathrm{m}}, \varepsilon_{\mathrm{o}}=\varepsilon_{\mathrm{c}} / \varepsilon_{\mathrm{m}}, \varepsilon_{\mathrm{c}}=1+4 \pi \gamma / v_{0}$ is the dielectric function of the HITC coating layer, $\rho_{v}=V_{i} n_{v}{ }^{\circ}$ $V_{0} n_{v}{ }^{i}, V_{i}=4 \pi a_{x} a_{y} a_{z} / 3, n_{v}{ }^{i}$ and $n_{v}{ }^{\circ}$ are the standard depolarization factors along the $v$ direction for the inner and outer spheroidal surfaces, respectively. We here, for simplicity, relate $b_{v}$ and $a_{v}$ according to $b_{v}=a_{v}+d$, where $d$ is the effective dye coating thickness (in the theory the inner and outer surfaces are confocal). The dye coating volume is $V_{\text {coat }}$ $=A d$, with $A$ being the surface area of the ellipsoid. $V_{\text {coat }}$ can also be determined by $V_{\text {coat }}=N v_{0}$, with $N$ and $v_{0}$ being the number of electromagnetically active dye molecules and the unit cell volume per molecule, respectively. ${ }^{30}$ The effective dye coating thickness is therefore related to the number of the active dye molecules according to $d=N v_{0} / A$. The scattering cross sections of the Au nanorod-HITC hybrid structures are calculated according to eqs 7 and 8 along the $x$ direction, because $\sigma_{y}{ }^{\text {scat }}$ and $\sigma_{z}^{\text {scat }}$ do not contribute to the longitudinal plasmon mode. In the calculations, the oscillator strength for the dye coating layer is $c_{0}=2.3$, the dielectric constant of the dye coating at large frequencies is $\varepsilon_{\mathrm{C}}(\omega \rightarrow \infty)$ $=1.77$, and the short semiaxes of the Au nanorods are $a_{y}=$ $a_{z}=10 \mathrm{~nm}$. The effective dye layer thickness $d$ is determined to be in the range of $0.7-1.5 \mathrm{~nm}$ according to the experimental scattering spectra. Very good agreements between the experimental and theoretical scattering spectra are obtained (Figure 3). The variation in the effective dye coating thickness reflects that the number of the adsorbed HITC molecules varies among different Au nanorods, which can be ascribed to that each nanorod has a slightly different environment in the agarose gel during HITC adsorption. In addition, the dye coating is assumed to have a uniform thickness around the ellipsoid surface in the analytic theory. It will be interesting to study the coupling behavior with nonuniformly adsorbed dye molecules.

The coupling energy diagram for the Au nanorods coated with the HITC adsorbate is further calculated using the two molecular resonances as the input parameters (Figure 4). When the plasmon resonance energy is smaller than the HITC's $M_{a}$ band, the resonance coupling causes a red shift in the plasmon band (Figure $4 \mathrm{~b}$ ). When the plasmon resonance energy is in between the $\mathrm{H}_{\mathrm{a}}$ band and $\sim 2.25 \mathrm{eV}$ (551
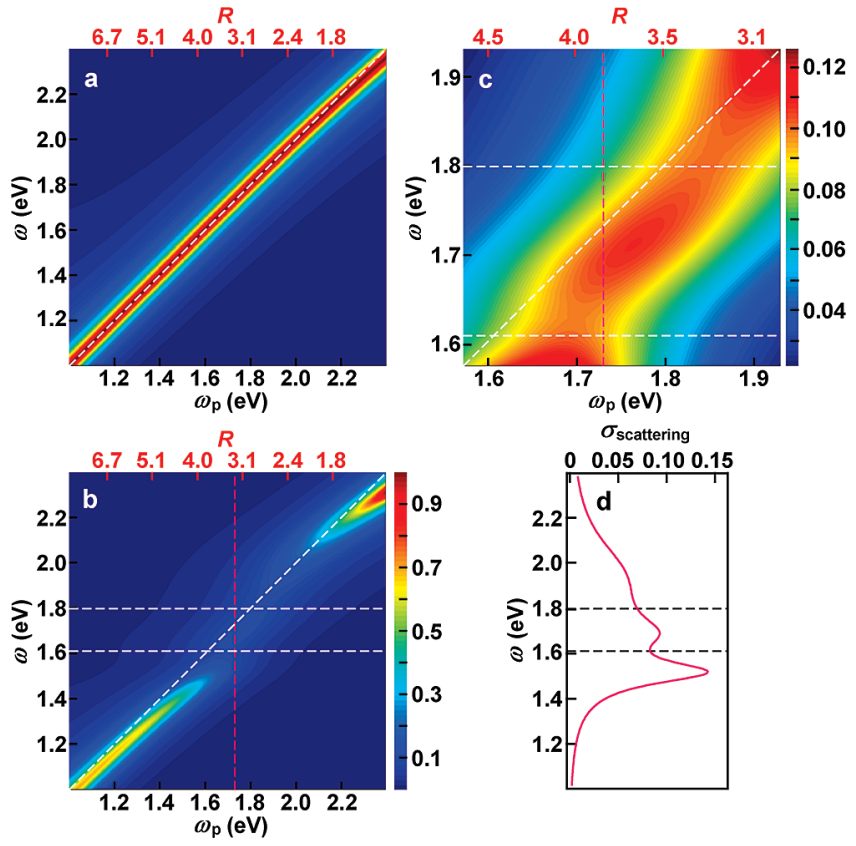

FIGURE 4. Plasmonic-molecular resonance coupling energy diagram between the Au nanorods and the HITC adsorbates. (a) Energy diagram for the Au nanorods without adsorbed HITC. (b) Coupling energy diagram for the Au nanorod-HITC hybrid nanostructures. The two horizontal lines represent the two molecular resonance energies of the HITC adsorbate. (c) Enlarged view of the coupling energy diagram, showing the presence of three bands. The intensities in the diagrams have been plotted at the logarithmic scale. The intensity scales are the same for (a) and (b). (d) Scattering spectrum extracted along the vertical line shown in (b) and (c). In the theoretical modeling, the effective oscillator strength $c_{0}$ for the HITC layer is set to be 2.3, and the effective HITC layer thickness $d$ is set to be $1.4 \mathrm{~nm}$.

$\mathrm{nm}$ ), the plasmon band shifts to higher energies upon the resonance coupling. These results are in good agreement with the experimental observations (Figure 3). When the plasmon resonance energy is larger than $\sim 2.25 \mathrm{eV}$, the plasmon band shifts to lower energies upon the adsorption of the dye molecules. In this energy region, because the plasmon resonance energy is far away from the molecular resonance energies, the resonance coupling is very weak. The dye-coating layer acts solely as a dielectric medium. The increase in the refractive index arising from the dielectric layer causes the red shift in the plasmon resonance. The same index effect also occurs when the plasmon resonance energy is much smaller than the $\mathrm{M}_{\mathrm{a}}$ band. When the plasmon band is in between the $\mathrm{M}_{\mathrm{a}}$ and $\mathrm{H}_{\mathrm{a}}$ band, it splits into three branches, as revealed by the magnified energy diagram in Figure 4c. The scattering spectra obtained by extracting the data points along the vertical direction clearly show three peaks when the plasmon band is in between the two molecular resonance bands (Figure 4d). The appearance of three branches in the calculated coupling energy diagram is in agreement with our scattering spectral measurements (Figure 2a), indicating the formation of three hybridized states (Figure 2b). In comparison, in the resonance coupling involving a single molecular resonance band, an anticrossing 
behavior between the plasmonic and molecular bands is usually observed, and the original plasmon band splits into two branches. ${ }^{15,17,18,29}$ These results suggest that the resonance coupling behavior is highly dependent on the molecular resonances of the dyes adsorbed on metal nanocrystals and point to a way for manipulating the plasmonic resonances with different dyes and their aggregates.

The resonance coupling can be tuned in real time by removing the molecular resonances. Because HITC can be photodecomposed by laser irradiation at $488 \mathrm{~nm}$, an argon ion laser (Spectra-Physics) was employed for the irradiation on the individual nanorod-HITC hybrid nanostructures embedded in the agarose gel films. The laser beam tuned at $20 \mathrm{~mW}$ was reflected by a dichroic mirror to the back aperture of the objective. The irradiation laser was blocked every time when a dark-field measurement was performed. Preprepared nanorod-HITC hybrid nanostructures were embedded in agarose gel films for the laser irradiation experiment. For the preparation of the gel films embedded with the hybrid nanostructures, HITC was first dissolved in water at $0.07 \mathrm{mM}$. Because HITC is difficult to be dissolved in water but readily in ethanol, the stock solutions of HITC at varying concentrations in 95/5 (v/v) mixtures of water and ethanol were made by first dissolving HITC in ethanol and then diluting the ethanolic solution with water. The concentration of HITC in water was calibrated using these stock solutions. An $800 \mu \mathrm{L}$ portion of the aqueous $0.07 \mathrm{mM}$ HITC solution was mixed with $0.2 \mathrm{~mL}$ of the 2 nM PSS-coated $\mathrm{Au}$ nanorod solution to produce nanorod-HITC hybrid nanostructures. After being kept undisturbed for $30 \mathrm{~min}, 150 \mu \mathrm{L}$ of the hybrid nanostructure solution was added into a glass beaker containing $10 \mathrm{mg}$ of agarose and $5 \mathrm{~mL}$ of water. The beaker was placed in a water bath at $80^{\circ} \mathrm{C}$, and the mixture solution was kept under mild stirring. While being kept in the water bath, the gel solution was dip-coated onto clean glass slides at a speed of $1.5 \mathrm{~cm} \mathrm{~min}^{-1}$. The resultant gel films were kept horizontally for 30 min before the laser irradiation and optical measurements. The gel films are 200-230 nm thick. They are denser and therefore more stable under the laser irradiation than those made by simple dropcasting

Figure 5 a shows the scattering spectra recorded on a hybrid nanostructure as a function of the laser irradiation time (see also Figure S5, Supporting Information). The scattering peak shifts to higher energies and becomes stronger gradually. After $\sim 20$ min of irradiation, the peak shift and intensity increase are ceased, suggesting that all of the adsorbed HITC molecules are photodecomposed. The decoupling process can be modeled analytically (Figure $5 \mathrm{~b}$ ), where the aspect ratio $R$ of the nanorod is determined from the scattering spectrum taken after the complete photodecomposition of HITC and the effective oscillator strength $c_{0}$ and the initial dye layer thickness $d$ are determined from the scattering spectrum taken before the irradiation. The reduced $c_{0}$ in Figure 5 compared to that in Figure 3 is
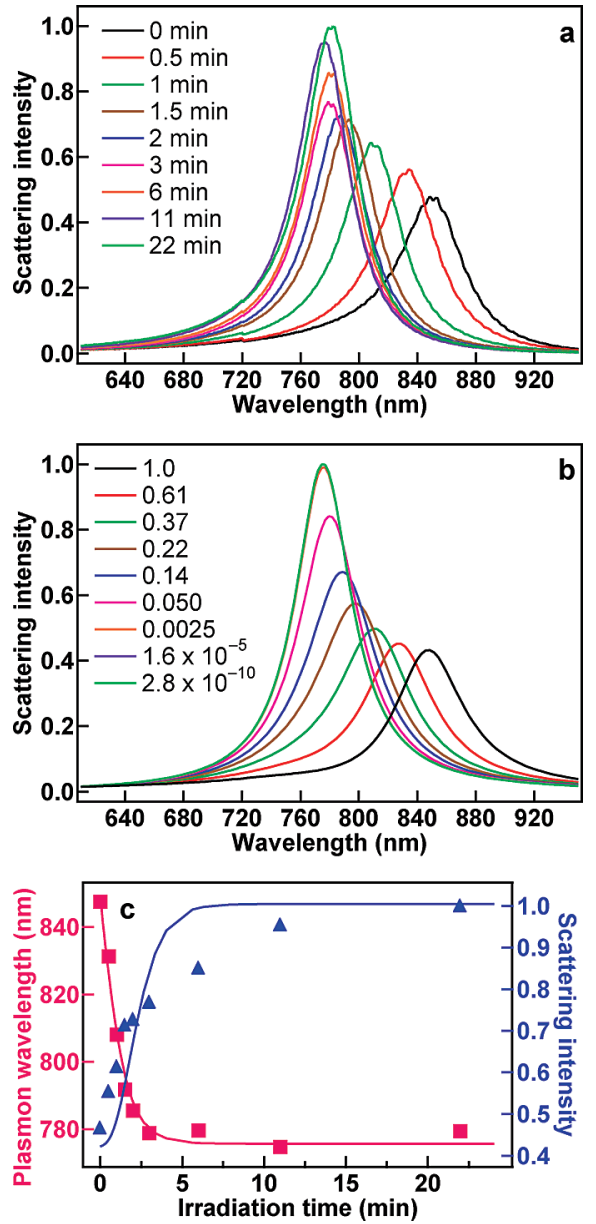

FIGURE 5. Tuning the resonance coupling by photodecomposition. (a) Scattering spectral evolution of a single Au nanorod-HITC hybrid nanostructure as a function of the laser irradiation time. The spectra have been normalized against the strongest one. (b) Theoretical modeling. The labels indicate the fraction of the active HITC molecules that are left after varying periods of the laser irradiation time. In the modeling of the scattering spectra, the effective dye layer thickness was calculated according to $d(t)=N(t) v_{0} / A$, with $t$ being the laser irradiation time. The initial effective dye layer thickness is $d(0)=2.8 \mathrm{~nm}$, and the effective oscillator strength $c_{0}$ is fixed at 0.63. (c) Dependence of the experimental scattering peak wavelength (red squares) and intensity (blue triangles) on the irradiation time. The lines are from the modeling.

ascribed to the different preparation methods for the hybrid nanostructures. During the irradiation, the effective oscillator strength $c_{0}$ is fixed and the effective dye layer thickness $d$ is found to decrease exponentially. Because the effective dye coating thickness is proportional to the number of the electromagnetically active dye molecules for the same nanorod, the reduction in the effective dye coating thickness during the laser irradiation indicates that the adsorbed HITC molecules are gradually photodecomposed. The experimental and theoretical variations of the plasmon shift and peak intensity versus the laser irradiation time are in good agreement (Figure $5 \mathrm{c}$ ). We also note that the HITC coverage on the Au nanorod at the late stage of the irradiation is probably less than one monolayer. The scattering spectra at the late stage can still be modeled by setting the effective dye layer 
thickness $d$ to be very small. These results suggest that the contribution of each dye molecule or aggregate to the resonance coupling is additive and that the analytic theory used for modeling the resonance coupling is very robust. In addition, the decoupling-induced blue shift in the scattering peak reaches $70 \mathrm{~nm}$ (Figure 5a). Such a large shift indicates the potential for developing ultrasensitive sensing devices on the basis of the plasmonic-molecular resonance coupling.

In summary, a direct measurement of the plasmonicmolecular resonance coupling on single Au nanorods has been demonstrated by embedding Au nanorods in agarose gel films. The gel films provide an interconnected porous network that can not only allow Au nanorods to be spatially isolated for single-particle imaging and spectroscopy but also carry out chemical and physical processes on the individual Au nanorods in liquid environments. The resonance coupling between the Au nanorods and HITC adsorbates has been investigated as a function of the nanorod plasmon resonance wavelength. The plasmon band is observed to split into three bands when it is in between the two molecular resonance bands of HITC. An analytic theory has been employed to model the resonance coupling between the Au nanorods and HITC. An excellent agreement between the experimental and theoretical scattering spectra has been obtained. The real-time tunability of the resonance coupling has been further demonstrated by photodecomposition and modeled theoretically.

Acknowledgment. We are grateful for financial support of this work from CUHK Research Excellence Award 20082009 (Project Code: 4411435), the Knut and Alice Wallenberg Foundation, and National Natural Science Foundation of China (Project Code: 20828001). We also thank F. Jäckel and J. Feldmann for helpful discussions.

Supporting Information Available. The extinction spectra of the gel films containing the Au nanorods and the hybrid nanostructures, the schematic of the dark-field scattering setup, the fluorescence quenching, a series of scattering images showing the tracking of the Au nanorods embedded in the gel film along the vertical direction, and the scattering and spectral images acquired during the laser irradiation. This material is available free of charge via the Internet at http://pubs.acs.org

\section{REFERENCES AND NOTES}

(1) Anger, P.; Bharadwaj, P.; Novotny, L. Phys. Rev. Lett. 2006, 96, 113002 .

(2) Kühn, S.; Håkanson, U.; Rogobete, L.; Sandoghdar, V. Phys. Rev. Lett. 2006, 97, No. 017402

(3) Tam, F.; Goodrich, G. P.; Johnson, B. R.; Halas, N. J. Nano Lett. 20077, 496-501
(4) Chen, Y.; Munechika, K.; Ginger, D. S. Nano Lett. 2007, 7, 690696.

(5) Zhang, J.; Fu, Y.; Chowdhury, M. H.; Lakowicz, J. R. Nano Lett. 2007, 7, 2101-2107.

(6) Bek, A.; Jansen, R.; Ringler, M.; Mayilo, S.; Klar, T. A.; Feldmann, J. Nano Lett. 2008, 8, 485-490.

(7) Dulkeith, E.; Morteani, A. C.; Niedereichholz, T.; Klar, T. A.; Feldmann, J.; Levi, S. A.; van Veggel, F. C. J. M.; Reinhoudt, D. N.; Möller, M.; Gittins, D. I. Phys. Rev. Lett. 2002, 89, 203002.

(8) Schneider, G.; Decher, G.; Nerambourg, N.; Praho, R.; Werts, M. H. V.; Blanchard-Desce, M. Nano Lett. 2006, 6, 530-536.

(9) Liu, G. L.; Long, Y.-T.; Choi, Y.; Kang, T.; Lee, L. P. Nat. Methods 2007, 4, 1015-1017.

(10) Kometani, N.; Tsubonishi, M.; Fujita, T.; Asami, K.; Yonezawa, Y. Langmuir 2001, 17, 578-580.

(11) Wiederrecht, G. P.; Wurtz, G. A.; Hranisavljevic, J. Nano. Lett. 2004, 4, 2121-2125.

(12) Zhang, W.; Govorov, A. O.; Bryant, G. W. Phys. Rev. Lett. 2006, 97,146804

(13) Zhao, J.; Jensen, L.; Sung, J. H.; Zou, S. L.; Schatz, G. C.; Van Duyne, R. P. J. Am. Chem. Soc. 2007, 129, 7647-7656.

(14) Uwada, T.; Toyota, R.; Masuhara, H.; Asahi, T. J. Phys. Chem. C 2007, 111, 1549-1552.

(15) Wurtz, G. A.; Evans, P. R.; Hendren, W.; Atkinson, R.; Dickson, W.; Pollard, R. J.; Zayats, A. V. Nano Lett. 2007, 7, 1297-1303.

(16) Kelley, A. M. Nano Lett. 2007, 7, 3235-3240.

(17) Ni, W. H.; Yang, Z.; Chen, H. J.; Li, L.; Wang, J. F. J. Am. Chem. Soc. 2008, 130, 6692-6693.

(18) Fofang, N. T.; Park, T.-H.; Neumann, O.; Mirin, N. A.; Nordlander, P.; Halas, N. J. Nano Lett. 2008, 8, 3481-3487.

(19) Yan, J.-Y.; Zhang, W.; Duan, S. Q.; Zhao, X.-G.; Govorov, A. O. Phys. Rev. B 2008, 77, 165301

(20) Ringler, M.; Schwemer, A.; Wunderlich, M.; Nichtl, A.; Kürzinger, K.; Klar, T. A.; Feldmann, J. Phys. Rev. Lett. 2008, 100, 203002.

(21) Sönnichsen, C.; Franzl, T.; Wilk, T.; von Plessen, G.; Feldmann, J. Phys. Rev. Lett. 2002, 88, No. 077402 .

(22) Ni, W. H.; Kou, X. S.; Yang, Z.; Wang, J. F. ACS Nano 2008, 2, 677-686.

(23) Tsung, C.-K.; Kou, X. S.; Shi, Q. H.; Zhang, J. P.; Yeung, M. H.; Wang, J. F.; Stucky, G. D. J. Am. Chem. Soc. 2006, 128, $5352-$ 5353.

(24) Kou, X. S.; Zhang, S. Z.; Yang, Z.; Tsung, C.-K.; Stucky, G. D.; Sun, L. D.; Wang, J. F.; Yan, C. H. J. Am. Chem. Soc. 2007, 129, 64026404

(25) Herz, A. H. Adsorption of sensitizing dyes to silver halides In the Theory of the Photographic Process; James, T. H., Ed.; Macmillan Publishing Co., Inc.: New York, 1977.

(26) Pastoriza-Santos, I.; Pérez-Juste, J.; Liz-Marzán, L. M. Chem. Mater. 2006, 18, 2465-2467

(27) Sturmer, D. M.; Heseltine, D. W. Sensitizing and desensitizing dyes. In the Theory of the Photographic Process; James, T. H., Ed.; Macmillan Publishing Co., Inc.: New York, 1977.

(28) Ambjörnsson, T.; Mukhopadhyay, G. J. Phys. A: Math. Gen. 2003, 36, 10651-10665.

(29) Ambjörnsson, T.; Mukhopadhyay, G.; Apell, S. P.; Käll, M. Phys. Rev. B 2006, 73, No. 085412.

(30) Ambjörnsson, T.; Apell, S. P.; Mukhopadhyay, G. Phys. Rev. E 2004, 69, No. 031914

(31) Lee, K.-S.; El-Sayed, M. A. J. Phys. Chem. B 2005, 109, $20331-$ 20338.

(32) Bohren, C. F.; Huffman, D. R. Absorption and Scattering of Light by Small Particles; John Wiley \& Sons: New York, 1983. 